\title{
Patient-reported outcomes in type 2 diabetes mellitus: patients' and primary care physicians' perspectives in the Spanish health care system
}

This article was published in the following Dove Press journal:

Patient Preference and Adherence

8 October 2015

Number of times this article has been viewed

\author{
Josep Franch-Nadal ${ }^{1,2}$ \\ Elena Labrador Barba ${ }^{3}$ \\ M Carmen Gómez-García ${ }^{4}$ \\ Pilar Buil-Cosiales ${ }^{5}$ \\ José Manuel Millaruelo 6 \\ María Luisa Orera Peña ${ }^{3}$ \\ 'AEP Raval Sud, Barcelona, ${ }^{2}$ Center \\ for Biomedical Research Network of \\ Diabetes and Associated Metabolic \\ Diseases (CIBERDEM), Madrid, \\ ${ }^{3}$ Established Pharmaceuticals Division, \\ Mylan EPD, Madrid, ${ }^{4}$ Centro de Salud \\ Velez Norte, Málaga, ${ }^{5}$ Centro de Salud \\ Azpilagana, Pamplona, ${ }^{6}$ Centro de \\ Salud Torrero La Paz, Zaragoza, Spain
}

Correspondence: Elena Labrador Barba Established Pharmaceuticals Division, Mylan EPD, Avenida Burgos, 9I, 28050 Madrid, Spain

Tel +34 9l 3373350

Email elena.labrador@mylan.com
Objective: Understanding patients' and physicians' perceptions of type 2 diabetes mellitus (T2DM) management and treatment has important implications for diabetes care, allowing the identification of clinical practice issues that could be improved, leading to patients' better understanding of the illness and, consequently, healthier self-management behaviors. The objective of this study was to identify differences between physicians' and T2DM patients' perceptions related to health status, patient-reported outcomes assessments, and T2DM management and treatment, in routine clinical practice in Spain.

Methods: This was an observational, cross-sectional study including 1,012 T2DM patients and 974 physicians from 47 and 52 Spanish provinces, respectively. An electronic structured self-administered questionnaire containing 17 questions was designed aiming to address both physicians' and patient's perceptions on overall T2DM health status and patient-reported outcomes.

Results: T2DM patients perceived a worse health status (40\% reported having a "good" and $38 \%$ a "neither good nor bad" health status) compared with physicians' perceptions (77\% thought patients had a "good" health status). Most patients answered being "satisfied" or "neither satisfied nor unsatisfied" with the given information, while physicians considered that patients were "satisfied" or "very satisfied" with the information for self-monitoring blood glucose and treatment administration. Fifty-seven percent of patients reported that medical recommendations were "important", while 58\% of physicians considered it as "very important". Fifty-three percent of patients perceived that their current T2DM treatment suited their preferences "quite a lot", and this was lower than the proportion of physicians $(69 \%)$ that believed this for their patients. Additionally, a lower percentage of patients (53\%) than physicians (79\%) believed that their treatment improved their health-related quality of life "quite a lot". All differences between patients and physicians were statistically significant $(P<0.001)$.

Conclusion: Patients and physicians demonstrate different views concerning all questions related to T2DM health status and diabetes management and treatment (information, recommendations, satisfaction, and preferences).

Keywords: T2DM, PROs, health-related quality of life, HRQoL, preferences, adherence, treatment satisfaction, perception

\section{Introduction}

Worldwide, the 2013 prevalence of diabetes mellitus (DM) was estimated at 8.3\%, affecting 387 million people and producing 5.1 million deaths. ${ }^{1}$ This prevalence is expected to increase up to 439 million adults by $2030 .^{2}$ In Spain, DM prevalence among adults is $13.8 \%$ (95\% confidence interval: $10 ; 15)$, with up to $6 \%$ of the population remaining underdiagnosed ${ }^{3}$ and having a mortality risk up to three times greater than 
the general population. ${ }^{4}$ Type 2 DM (T2DM) accounts for $85 \%-95 \%$ of all diabetes in developed countries, ${ }^{1}$ and it is associated with a two to four times greater probability of cardiovascular disease than in the general population, as well as an increased mortality risk. ${ }^{5,6}$

It is widely recognized that an appropriate management of hyperglycemia can lead to an achievement of optimal diabetes control, thereby reducing the risk of cardiovascular events by approximately $50 \%{ }^{7}$ However, the micro- and macrovascular complications associated with long-term disease continue to be the main factor for the social and economic burden of T2DM. ${ }^{8}$ In addition to clinical and economic aspects, T2DM may have a direct influence on daily personal, family, social, and working life, affecting patients' functional capacity and health-related quality of life (HRQoL). ${ }^{9}$

Therefore, T2DM is considered a complex chronic disease, which requires continuous medical care with multifactorial risk reduction strategies beyond glycemic control. ${ }^{10}$ In addition, the successful management of diabetes should include the active behavioral involvement of patients. Patients diagnosed with T2DM need to implement certain habits that require self-commitment and responsibility (eg, blood glucose monitoring in diabetes; adherence to medication, etc). ${ }^{11,12}$ For this purpose, patient education regarding glucose monitoring, injection techniques, and lifestyle interventions, focusing on diet and on the importance of physical activity, should be integrated into the treatment program. The involvement of patients is crucial for the management of T2DM, for the prevention of acute complications, and for the reduction of long-term complications. ${ }^{10,13}$ In Spain, where T2DM patients are mainly managed in the primary care (PC) setting, ${ }^{14}$ a patient-physician partnership is key to achieving optimal treatment results and to improving patients' empowerment and disease management. ${ }^{11}$

Moreover, patients' involvement in decision making may improve adherence to therapy. ${ }^{13}$ When patients feel that their disease perspective and experience (patient-reported outcomes [PROs]) are taken into account by PC physicians, they increase their medication adherence and cooperate more actively with lifestyle recommendations, improving personal satisfaction, clinical results, ${ }^{11}$ and overall quality of care. ${ }^{15}$ In this study, it is proposed that the knowledge from both patients' and health care professionals' perspectives and experience in real-life clinical practice may have important implications for diabetes care. Recognizing such information allows the identification of practice issues that could be improved or the potential introduction of changes in health care plans. ${ }^{16}$ Nevertheless, it has been difficult to find in the literature studies that elicit patient self-perceived
HRQoL added to their perception of PROs assessment, and that compare patients' perceptions and beliefs with those of PC physicians.

Therefore, to the authors' knowledge, this is the first study specifically designed to identify differences between physicians' and T2DM patients' perceptions regarding health status, PROs evaluation, and T2DM management and treatment, from the Spanish National Healthcare System (NHS) perspective.

\section{Research design and methods}

An observational, cross-sectional study was performed. The study protocol was approved by the Clinical Research Ethics Committee of the Hospital Universitario Puerta de Hierro (Majadahonda, Madrid). All participants in the study received information and gave their consent to participate prior to their inclusion in the study.

\section{Participants}

Sample size estimation was calculated based on the 2013 Spanish adult population $(34,581,569),{ }^{17}$ published data on the prevalence of T2DM patients $(10 \%-15 \%),{ }^{3}$ and the number of PC physicians practicing in the Spanish NHS $(28,675) .{ }^{18}$ Applying the maximum variability standard criteria with a $99 \%$ confidence level and $4 \%$ precision for both groups, the sample size required was estimated at 1,012 T2DM patients and 974 PC physicians.

\section{Patients}

Eligible participants were T2DM patients at least 18 years old who were able to adequately answer the study questions. Patient selection was carried out with the support of a FEDE (Federación de Diabéticos Españoles [Spanish Diabetic Patients Federation]). Members of the different societies belonging to the FEDE from 52 Spanish provinces were invited to participate. The recruitment process was conducted from February to June 2014, until the desired sample size was achieved.

\section{Physicians}

PC physicians from 52 Spanish provinces were randomly selected and invited to take part in the study in collaboration with the Spanish Physician Association RedGDPs (Red de Grupos de Estudio de la Diabetes en Atención Primaria de la Salud). Physicians were eligible to participate if they practiced in the Spanish public National Health care System (NHS) and if they had been practicing their profession for at least 5 years post-training residency. The recruitment process was also conducted from February to June 2014, until the desired sample size was achieved. 


\section{Survey design}

An ad hoc electronic self-administered questionnaire containing 17 questions was developed for both patients and physicians, respectively, based on the information obtained from a literature review. The survey was structured in three parts: 1) two questions about the current health status of T2DM patients; 2) five questions related to PROs evaluation in T2DM (HRQoL and treatment persistence, adherence, satisfaction, and preferences); and 3) ten questions about T2DM management and treatment. All questions were rated using a five-point Likert scale (Table 1). In all cases, the answers corresponded to the self-perception of T2DM patients and the perception of physicians about their T2DM population, respectively. In order to facilitate patients' participation, they could also answer the questionnaire by phone in case of unavailability of Internet access.

\section{Study variables}

Sociodemographic variables were collected for both populations. Specifically, for T2DM patients, the variables collected were age, sex, place of residence, educational level, and employment status. Moreover, patients informed about the following clinical variables: time since T2DM diagnosis, treatment type (oral, injected, combined therapy), and comorbidities (hypertension, hypercholesterolemia, and obesity) and their treatment. Physicians reported the following variables: age, sex, health care center area, and approximate number of T2DM patients attending per month.

\section{Analysis}

A descriptive analysis was performed including all participants with completed responses. Absolute and relative frequencies were calculated for qualitative variables. Measurements of central tendency and dispersion were reported for quantitative variables. Scores distribution obtained for each question was compared between groups applying the chi-square test. For all statistical tests, $P<0.05$ was considered significant. SPSS version 19.0 was used for data analysis.

\section{Results}

\section{Description of the participants}

Of the 6,747 patients who were invited to participate in the survey, a total of $1,012(15 \%)$ T2DM patients from 47 different Spanish provinces participated in the study. On the other hand, of the 1,021 PC physicians invited to participate in the survey, a total of $974(95.4 \%)$ responded to

Table I Ad-hoc self administered survey used in the study

\begin{tabular}{|c|c|}
\hline Assessed aspects & Questions (five-point Likert scale) \\
\hline \multirow[t]{2}{*}{ I. Health status } & I. Patient current health status ${ }^{\mathrm{a}}$ \\
\hline & 2. How much does the T2DM affect your current health status ${ }^{b}$ \\
\hline \multirow{5}{*}{$\begin{array}{l}\text { 2. PROs: HRQoL and treatment persistence, } \\
\text { adherence, satisfaction, and preferences }\end{array}$} & 3. Frequency of PROs evaluation by physicians ${ }^{c}$ \\
\hline & 4. Importance given to the PROs evaluation by physicians ${ }^{d}$ \\
\hline & 5. Frequency of changes in T2DM treatment to improve PROs ${ }^{c}$ \\
\hline & 6. Frequency of changes in T2DM treatment to suit patient preferences ${ }^{c}$ \\
\hline & 7. Physician-patient relationship ${ }^{e}$ \\
\hline \multirow[t]{10}{*}{ 3. Diabetes management and treatment } & $\begin{array}{l}\text { 8. Frequency with which nurses provide information about T2DM, SMBG, and treatment } \\
\text { administration techniques (oral or injectable) }\end{array}$ \\
\hline & $\begin{array}{l}\text { 9. Patient satisfaction with the information provided by nurses about T2DM, SMBG, and treatment } \\
\text { administration techniques (oral or injectable) }{ }^{g}\end{array}$ \\
\hline & $\begin{array}{l}\text { 10. Frequency with which patients follow physicians' recommendations about routine SMBG, } \\
\text { routine medical visits, diet, exercise, oral treatment, and injectable treatment }{ }^{f}\end{array}$ \\
\hline & $\begin{array}{l}\text { II. Patient satisfaction with physicians' recommendations about routine SMBG, routine medical } \\
\text { visits, diet, exercise, oral treatment, and injectable treatment }{ }^{g}\end{array}$ \\
\hline & $\begin{array}{l}\text { 12. The extent to which physicians' recommendations about routine SMBG, routine medical visits, } \\
\text { diet, exercise, oral treatment, and injectable treatment allow T2DM control }{ }^{b}\end{array}$ \\
\hline & 13. Importance given to the physicians' recommendations ${ }^{d}$ \\
\hline & 14. Frequency with which comorbidities are considered by physicians when deciding the T2DM treatment ${ }^{f}$ \\
\hline & I5. The extent to which T2DM treatment suits patient preferences ${ }^{\mathrm{b}}$ \\
\hline & 16. The extent to which T2DM treatment improves patient HRQoL ${ }^{b}$ \\
\hline & $\begin{array}{l}\text { 17. Importance of new technologies (PDAs, cell phone applications, online information, etc) for } \\
\text { diabetes self-management }{ }^{b}\end{array}$ \\
\hline
\end{tabular}

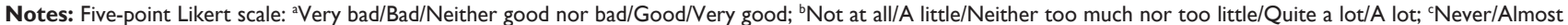

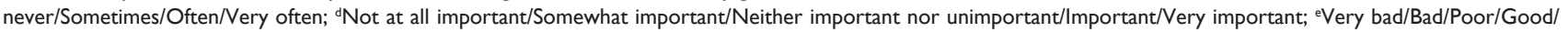
Very good; ${ }^{\mathrm{N}} \mathrm{Never/Almost}$ never/Sometimes/Frequently/Always; ${ }^{\mathrm{g} N o t}$ at all satisfied/Somewhat unsatisfied/Neither satisfied nor unsatisfied/Satisfied/Very satisfied.

Abbreviations: HRQoL, health-related quality of life; PDAs, personal digital assistants; PROs, patient-reported outcomes; SMBG, self-management of blood glucose; T2DM, type 2 diabetes mellitus. 
the questionnaires, distributed among 52 Spanish provinces. The final sample for this analysis was therefore composed of 1,012 patients and 974 independent physicians, being 1.04 patients included in the study per physician.

The patients' mean age was 54.2 (standard deviation [SD]: 11.2) years, and approximately half of the studied subjects were males (51\%). Only $26 \%(n=266)$ of participants had college education and $44 \%(n=445)$ were retired at the time of the study. The mean time since T2DM diagnosis was 11.3 (SD: 9.7) years. Ninety percent of patients $(n=917)$ received prescribed medication for their diabetes $(51.2 \%$ $[\mathrm{n}=518]$ were treated with oral medication only, $15.3 \%$ $[\mathrm{n}=155]$ with injectable treatment alone, and 24.1\% [n=244] received both oral and injected medications).

Participant physicians had a mean age of 52.4 (SD: 7.8) years, being $66.3 \%(n=646)$ male. Overall, 39\% $(n=380)$ of physicians reported seeing one to $50 \mathrm{~T} 2 \mathrm{DM}$ patients per month, $36 \%(n=351)$ indicated 51 to 100 , and $25 \%(n=243)$ estimated more than 100 visits per month. Patients' and physicians' sociodemographic and clinical variables are described in Table 2.

Table 2 Sociodemographic and clinical characteristics of the T2DM patients and physicians

\begin{tabular}{|c|c|c|}
\hline Characteristics & T2DM patients $n=I, 0 I 2$ & Physicians n=974 \\
\hline Age (years), mean (SD) & $54.2(11.2)$ & $52.4(7.8)$ \\
\hline Male, n (\%) & $519(5 \mathrm{I})$ & $646(66.3)$ \\
\hline \multicolumn{3}{|l|}{ Place of residence, $n(\%)$} \\
\hline Rural $(<5,000$ inhabitants $)$ & $99(10)$ & $182(19)$ \\
\hline Semi-urban $(5,000-19,999$ inhabitants $)$ & $223(22)$ & $252(26)$ \\
\hline Urban ( $\geq 20,000$ inhabitants $)$ & $690(68)$ & $540(55)$ \\
\hline \multicolumn{3}{|l|}{ Educational level, n (\%) } \\
\hline No education & $93(9)$ & - \\
\hline Primary school & $275(27)$ & - \\
\hline High school & $220(22)$ & - \\
\hline Job training & $158(16)$ & - \\
\hline College studies (undergraduate) & $226(22)$ & - \\
\hline College studies (graduate) & $40(4)$ & - \\
\hline \multicolumn{3}{|l|}{ Employment status, $n$ (\%) } \\
\hline Worker & $289(29)$ & - \\
\hline Freelance & $64(6)$ & - \\
\hline Incapacity & $20(2)$ & - \\
\hline Student & $4(0.4)$ & - \\
\hline Unemployed & $83(8)$ & - \\
\hline Retired & $445(44)$ & - \\
\hline Housework & $105(10)$ & - \\
\hline Other & $2(0.2)$ & - \\
\hline Time from diagnosis (years), mean (SD) & $11.3(9.7)$ & - \\
\hline Time duration (years), mean (SD) & $10.7(9.6)$ & - \\
\hline \multicolumn{3}{|l|}{ Administration route, $\mathrm{n}(\%)$} \\
\hline Oral & $762(75)$ & - \\
\hline Injectable & $400(40)$ & - \\
\hline \multicolumn{3}{|l|}{ Comorbidities, n (\%) } \\
\hline \multicolumn{3}{|l|}{ Hypertension } \\
\hline Diagnosis & $482(48)$ & - \\
\hline Pharmacological treatment & $424(42)$ & - \\
\hline \multicolumn{3}{|l|}{ Hypercholesterolemia } \\
\hline Diagnosis & $663(66)$ & - \\
\hline Pharmacological treatment & $378(37)$ & - \\
\hline \multicolumn{3}{|l|}{ Hypertriglyceridemia } \\
\hline Diagnosis & $515(5 \mathrm{I})$ & - \\
\hline Pharmacological treatment & $164(16)$ & - \\
\hline \multicolumn{3}{|l|}{ Obesity } \\
\hline Diagnosis & $4 I I(4 I)$ & - \\
\hline Pharmacological Treatment & $56(6)$ & - \\
\hline \multicolumn{3}{|l|}{ Medical specialty, n (\%) } \\
\hline Primary care & - & $959(98.5)$ \\
\hline Other & - & $15(1.5)$ \\
\hline T2DM patients visited per month, mean (SD) & - & $91(74)$ \\
\hline
\end{tabular}

Abbreviations: T2DM, type 2 diabetes mellitus; SD, standard deviation. 


\section{Questionnaire scores}

Statistically significant differences between patients' and physicians' scores were found in all the included questions $(P<0.001)$.

\section{Health status questions}

The questionnaire scores showed that T2DM patients perceived a worse health status compared with physicians' perceptions. Seventy-seven percent of physicians answered that their patients had a "good" health status, while most patients considered their health status was "good" (40\%) or "neither good nor bad" (38\%).

On the other hand, patients thought that T2DM had a lower impact on their current health status compared with physicians, since most patients answered that T2DM affected their health status "neither too much nor too little" (38\%), while $74 \%$ of physicians thought that T2DM affected patients' health status "a lot".

\section{PROs questions}

Table 3 reflects the relative importance given by both patients and physicians to PROs assessment (HRQoL and treatment persistence, adherence, satisfaction, and preferences) and the relative importance given by physicians to the impact of PROs on T2DM treatment and management.

In general, most patients reported that their doctors assessed PROs "sometimes" or "often". However, physicians mainly indicated that they "often" or "very often" asked their

Table 3 PROs questions*

\begin{tabular}{|c|c|c|c|c|c|c|}
\hline & \multicolumn{6}{|c|}{ Frequency of PROs evaluation by Phy, \% (95\% CI) } \\
\hline & Group & Never & Almost never & Sometimes & Often & Very often \\
\hline \multirow[t]{2}{*}{ HRQoL } & Pat $(n=1,012)$ & $7(5 ; 8)$ & II $(9 ;$ I3) & $28(25 ; 30)$ & $40(37 ; 43)$ & $15(13 ; 17)$ \\
\hline & Phy $(n=974)$ & $0(0 ; 1)$ & $2(1 ; 3)$ & $21(19 ; 24)$ & $61(58 ; 65)$ & $15(13 ; 17)$ \\
\hline Treatment & Pat $(n=I, 0 \mid 2)$ & $14(11 ; 16)$ & $15(13 ; 18)$ & $30(27 ; 33)$ & $31(28 ; 34)$ & $10(8 ; 12)$ \\
\hline preferences & Phy $(n=974)$ & $0(0 ; 1)$ & $2(1 ; 3)$ & $18(16 ; 21)$ & $63(60 ; 66)$ & 17 (15; 19) \\
\hline Treatment & Pat $(n=I, 0 \mid 2)$ & $7(6 ; 9)$ & $7(6 ; 9)$ & $24(22 ; 27)$ & $43(40 ; 46)$ & $18(16 ; 21)$ \\
\hline persistence & Phy (n=974) & $0(0 ; 0)$ & $0(0 ; 3)$ & $8(6 ; 10)$ & $56(53 ; 59)$ & $36(33 ; 39)$ \\
\hline Treatment & Pat $(n=I, 012)$ & $7(5 ; 8)$ & $9(7 ;$ II) & $25(23 ; 28)$ & $42(39 ; 45)$ & $17(15 ; 20)$ \\
\hline adherence & Phy $(n=974)$ & $0(0 ; 0)$ & $0(0 ; 0)$ & $6(4 ; 7)$ & $49(45 ; 52)$ & $46(43 ; 49)$ \\
\hline Treatment & Pat $(n=I, 0 \mid 2)$ & $9(8 ;$ II) & $10(8 ; 12)$ & $28(26 ; 3 I)$ & $39(36 ; 42)$ & $13(11 ; 16)$ \\
\hline \multirow[t]{3}{*}{ satisfaction } & Phy $(n=974)$ & $0(0 ; 0)$ & $2(1 ; 3)$ & 17 (14; 19) & $59(55 ; 61)$ & $23(21 ; 26)$ \\
\hline & \multicolumn{6}{|c|}{ Importance given to the PROs evaluation by Phy, \% $(95 \% \mathrm{CI})$} \\
\hline & Group & $\begin{array}{l}\text { Not at all } \\
\text { important }\end{array}$ & $\begin{array}{l}\text { Somewhat } \\
\text { important }\end{array}$ & $\begin{array}{l}\text { Neither important } \\
\text { nor unimportant }\end{array}$ & Important & Very important \\
\hline \multirow[t]{2}{*}{ HRQoL } & Pat $(n=I, 0 I 2)$ & $\mathrm{I}(\mathrm{I} ; 2)$ & $3(2 ; 3)$ & $16(13 ; 18)$ & $47(44 ; 50)$ & $34(31 ; 40)$ \\
\hline & Phy $(n=974)$ & $0(0 ; 0)$ & $0(0 ; 1)$ & $\mathrm{I}(1 ; 2)$ & $52(49 ; 55)$ & $47(43 ; 50)$ \\
\hline Treatment & Pat $(n=I, 012)$ & $2(1 ; 2)$ & $4(3 ; 5)$ & $21(18 ; 23)$ & $52(49 ; 55)$ & $22(19 ; 24)$ \\
\hline preferences & Phy $(n=974)$ & $0(0 ; 0)$ & $\mathrm{I}(\mathrm{I} ; 2)$ & $12(10 ; 14)$ & $67(64 ; 70)$ & $20(17 ; 22)$ \\
\hline Treatment & Pat $(n=I, 0 \mid 2)$ & $\mathrm{I}(\mathrm{I} ; 2)$ & $4(3 ; 6)$ & $18(15 ; 20)$ & $5 I(48 ; 54)$ & $26(23 ; 29)$ \\
\hline persistence & Phy (n=974) & $0(0 ; 0)$ & $0(0 ; 0)$ & $2(1 ; 3)$ & $50(47 ; 53)$ & $48(45 ; 5 I)$ \\
\hline Treatment & Pat $(n=I, 0 \mid 2)$ & $2(I ; 3)$ & $3(2 ; 4)$ & $17(15 ; 20)$ & $53(50 ; 56)$ & $25(22 ; 27)$ \\
\hline adherence & Phy (n=974) & $0(0 ; 0)$ & $0(0 ; 1)$ & I (0; I) & $36(33 ; 39)$ & $63(60 ; 66)$ \\
\hline Treatment & Pat $(n=I, 0 \mid 2)$ & $\mathrm{I}(\mathrm{I} ; 2)$ & $4(2 ; 5)$ & 17 (15; 19) & $52(49 ; 55)$ & $26(23 ; 29)$ \\
\hline \multirow[t]{3}{*}{ satisfaction } & Phy $(n=974)$ & $0(0 ; 0)$ & $0(0 ; 1)$ & $5(4 ; 6)$ & $57(54 ; 60)$ & $38(35 ; 4 I)$ \\
\hline & \multicolumn{6}{|c|}{ Frequency of changes in T2DM treatment to improve PROs, \% (95\% CI) } \\
\hline & Group & Never & Almost never & Sometimes & Often & Very often \\
\hline \multirow[t]{2}{*}{ HRQoL } & Pat $(n=I, 0 \mid 2)$ & $12(10 ; 14)$ & $15(13 ; 17)$ & $35(32 ; 38)$ & $26(23 ; 29)$ & $13(11 ; 15)$ \\
\hline & Phy $(n=974)$ & $0(0 ; 1)$ & $4(3 ; 5)$ & $40(37 ; 43)$ & $47(44 ; 50)$ & $9(7 ; \mathrm{II})$ \\
\hline Treatment & Pat $(n=I, 0 \mid 2)$ & $15(13 ; 17)$ & $17(15 ; 20)$ & $31(29 ; 34)$ & $25(23 ; 28)$ & II (9; I3) \\
\hline persistence & Phy $(n=974)$ & $0(0 ; 0)$ & $3(2 ; 4)$ & $33(30 ; 36)$ & $5 \mathrm{I}(48 ; 54)$ & I3 (II; I5) \\
\hline Treatment & Pat $(n=I, 0 \mid 2)$ & $15(13 ; 17)$ & $16(13 ; 18)$ & $33(30 ; 36)$ & $25(22 ; 28)$ & II (9; I3) \\
\hline adherence & Phy $(n=974)$ & $0(0 ; 0)$ & $3(2 ; 4)$ & $24(2 \mathrm{I} ; 27)$ & $5 \mathrm{I}(48 ; 54)$ & $22(19 ; 24)$ \\
\hline Treatment & Pat $(n=I, 0 \mid 2)$ & $14(12 ; 16)$ & $19(17 ; 22)$ & $33(30 ; 36)$ & $24(2 I ; 26)$ & $10(8 ; 12)$ \\
\hline satisfaction & Phy (n=974) & $0(0 ; 1)$ & $4(3 ; 6)$ & $32(29 ; 35)$ & $50(46 ; 53)$ & $13(11 ; 16)$ \\
\hline
\end{tabular}

Note: $* P<0.001$ in all comparisons.

Abbreviations: $\mathrm{Cl}$, confidence interval; HRQoL, health-related quality of life; Pat, patients; Phy, physicians; PROs, patient-reported outcomes; T2DM, type 2 diabetes mellitus. 
patients about PROs. The estimated percentage of patients responding that physicians evaluated their treatment persistence "very often" was $18 \%$, compared to the $36 \%$ of physicians who considered they assessed patients' treatment persistence "very often". Interestingly, almost half of the physicians (46\%) thought they assessed treatment adherence "very often", while only $17 \%$ of patients agreed with this statement.

Added to this, both patients and physicians agreed on the importance of PROs for T2DM management; nevertheless, the proportion of physicians who considered their assessment "important" or "very important" was statistically significantly higher for all comparisons. As an example, most physicians (63\%) answered that treatment adherence was very important in order to achieve optimal clinical outcomes, while only $25 \%$ of patients considered this attribute of great importance for doctors. Moreover, $51 \%$ of the physicians included reported that they "often" changed treatment, aiming at improving treatment adherence, while only a quarter of patients $(25 \%)$ perceived that physicians changed treatment to improve their medication adherence.

In general, the majority of physicians reported that they "often" changed treatment in order to improve PROs, while most patients perceived that physicians only changed treatments "sometimes" in order to improve their PROs. Finally, although most participants answered that the patient-physician relationship was "good", while more physicians had a similar opinion about it (68\%) compared with patients (53\%).

\section{T2DM management and treatment questions}

\section{Information provided}

Regarding the information about treatment techniques and self-management provided to patients by nurses, the results in Table 4 show that the majority of patients answered that they received information about T2DM, self-monitoring of blood glucose (SMBG), and treatment administration techniques from nurses "sometimes" or "frequently", whereas physicians indicated that this information was given "frequently" or "always" to the patients. A high percentage of physicians perceived that nurses "always" assessed patients' abilities to check blood glucose levels $(38 \%)$ or trained them how to administrate their treatments (40\%), compared to $14 \%$ of patients who answered that they were "always" taught how to perform SMBG or received information regarding treatment dosage and administration (13\%).

In addition, most patients answered that they were "satisfied" or "neither satisfied nor unsatisfied" with the given information; however, patients' satisfaction was higher according to physicians, who considered that patients were "satisfied" or "very satisfied" with the information for SMBG and treatment administration.

\section{Recommendations}

Table 5 reports the frequency with which patients followed physicians' recommendations about routine SMBG or medical visits, diet, exercise, and oral treatments and the degree of satisfaction with these recommendations.

Table 4 T2DM management and treatment questions*

\begin{tabular}{|c|c|c|c|c|c|c|}
\hline & \multicolumn{6}{|c|}{ Frequency with which nurses provide information about the following topics, $\%(95 \% \mathrm{Cl})$} \\
\hline & Group & Never & Almost never & Sometimes & Frequently & Always \\
\hline \multirow[t]{2}{*}{ T2DM } & Pat $(n=I, 0 \mid 2)$ & $13(1 \mid ; 15)$ & $13(\mid 1 ; 15)$ & $30(27 ; 32)$ & $29(27 ; 32)$ & $15(13 ; 17)$ \\
\hline & Phy $(n=974)$ & $0(0 ; 1)$ & $4(3 ; 6)$ & $19(17 ; 22)$ & $48(44 ; 51)$ & $29(26 ; 32)$ \\
\hline \multirow[t]{2}{*}{ SMBG } & Pat $(n=I, 0 \mid 2)$ & $15(13 ; 17)$ & I3 (II; I5) & $30(27 ; 33)$ & $32(29 ; 35)$ & $14(12 ; 17)$ \\
\hline & Phy $(n=974)$ & I $(0 ; 1)$ & $2(I ; 3)$ & II $(9 ; 13)$ & $49(46 ; 52)$ & $38(34 ; 4 I)$ \\
\hline \multirow{4}{*}{$\begin{array}{l}\text { Treatment } \\
\text { administration } \\
\text { techniques }\end{array}$} & Pat $(n=I, 0 \mid 2)$ & $21(19 ; 24)$ & $14(12 ; 16)$ & $29(26 ; 32)$ & $27(25 ; 30)$ & $13(1 \mathrm{I} ; 15)$ \\
\hline & Phy $(n=974)$ & I $(0 ; 1)$ & $2(1.1 ; 3)$ & $14(12 ; 16)$ & $43(40 ; 46)$ & $40(37 ; 43)$ \\
\hline & \multicolumn{6}{|c|}{ Patient satisfaction with the information provided by nurses about the following topics, $\%(95 \% \mathrm{Cl})$} \\
\hline & Group & $\begin{array}{l}\text { Not at all } \\
\text { satisfied }\end{array}$ & $\begin{array}{l}\text { Somewhat } \\
\text { unsatisfied }\end{array}$ & $\begin{array}{l}\text { Neither satisfied } \\
\text { nor unsatisfied }\end{array}$ & Satisfied & Very satisfied \\
\hline \multirow[t]{2}{*}{ T2DM } & Pat $(n=I, 0 \mid 2)$ & $4(3 ; 6)$ & $7(5 ; 8)$ & $24(22 ; 27)$ & $47(44 ; 50)$ & $18(16 ; 20)$ \\
\hline & Phy $(n=974)$ & $\mathrm{I}(0 ; 2)$ & $4(3 ; 5)$ & $19(16 ; 21)$ & $60(57 ; 63)$ & $16(14 ; 19)$ \\
\hline \multirow[t]{2}{*}{ SMBG } & Pat $(n=1,012)$ & $5(4 ; 6)$ & $6(5 ; 7)$ & $24(21 ; 16)$ & $48(45 ; 5 I)$ & $18(15 ; 20)$ \\
\hline & Phy $(n=974)$ & $0(0 ; 1)$ & $3(2 ; 3)$ & II $(9 ; 13)$ & $61(58 ; 64)$ & $25(22 ; 27)$ \\
\hline \multirow{2}{*}{$\begin{array}{l}\text { Treatment } \\
\text { administration } \\
\text { techniques }\end{array}$} & Pat $(n=I, 0 \mid 2)$ & $6(5 ; 7)$ & $5(4 ; 7)$ & $29(26 ; 32)$ & $43(40 ; 46)$ & $17(14 ; 19)$ \\
\hline & Phy $(\mathrm{n}=974)$ & $0(0 ; 1)$ & $3(2 ; 4)$ & $12(10 ; 14)$ & $60(57 ; 63)$ & $25(23 ; 28)$ \\
\hline
\end{tabular}

Note: $* P<0.001$ in all comparisons.

Abbreviations: $\mathrm{Cl}$, confidence interval; Pat, patients; Phy, physicians; SMBG, self-management of blood glucose; T2DM, type 2 diabetes mellitus. 
Table 5 Physician recommendations*

\begin{tabular}{|c|c|c|c|c|c|c|}
\hline & \multicolumn{6}{|c|}{ Frequency with which patients follow physicians' recommendations about the following topics, \% $(95 \% \mathrm{Cl})$} \\
\hline & Group & Never & Almost never & Sometimes & Frequently & Always \\
\hline \multirow[t]{2}{*}{ Routine SMBG } & Pat $(n=I, 0 \mid 2)$ & $2(1 ; 2)$ & $3(2 ; 4)$ & $19(17 ; 21)$ & $4 \mathrm{I}(38 ; 44)$ & $36(33 ; 39)$ \\
\hline & Phy $(n=974)$ & $0(0 ; 0)$ & $I(0 ; 1)$ & $24(21 ; 27)$ & $65(61 ; 67)$ & II (9; 13) \\
\hline \multirow[t]{2}{*}{ Routine medical visits } & Pat $(n=|, 0| 2)$ & I $(0 ; 2)$ & $4(3 ; 5)$ & $17(14 ; 19)$ & $37(34 ; 40)$ & $4 \mathrm{I}(38 ; 44)$ \\
\hline & Phy $(n=974)$ & $0(0 ; 0)$ & $0(0 ; 0)$ & $14(12 ; 17)$ & $72(69 ; 75)$ & $14(12 ; 16)$ \\
\hline \multirow[t]{2}{*}{ Diet } & Pat $(n=|, 0| 2)$ & $2(1 ; 3)$ & $5(4 ; 7)$ & $25(22 ; 28)$ & $45(42 ; 48)$ & $23(20 ; 25)$ \\
\hline & Phy $(n=974)$ & $0(0 ; 1)$ & $15(12 ; 17)$ & $59(56 ; 62)$ & $23(21 ; 26)$ & $3(2 ; 4)$ \\
\hline \multirow[t]{2}{*}{ Exercise } & Pat $(n=I, 0 \mid 2)$ & $5(4 ; 6)$ & $12(10 ; 14)$ & $28(25 ; 3 I)$ & $35(32 ; 38)$ & $20(17 ; 22)$ \\
\hline & Phy $(n=974)$ & $0(0 ; 1)$ & $19(17 ; 22)$ & $59(56 ; 62)$ & $19(17 ; 22)$ & $2(1 ; 3)$ \\
\hline \multirow[t]{2}{*}{ Oral treatment } & Pat $(n=|, 0| 2)$ & $9(7 ; 11)$ & $2(1 ; 3)$ & II $(9 ; 13)$ & $31(28 ; 34)$ & $47(44 ; 50)$ \\
\hline & Phy $(n=974)$ & $0(0 ; 0)$ & $0(0 ; 0)$ & $4(3 ; 5)$ & $72(69 ; 75)$ & $24(21 ; 26)$ \\
\hline \multirow[t]{4}{*}{ Injectable treatment } & Pat $(n=|, 0| 2)$ & $34(31 ; 37)$ & $3(2 ; 5)$ & II $(9 ; 13)$ & $23(20 ; 26)$ & $29(27 ; 32)$ \\
\hline & Phy $(n=974)$ & $0(0 ; 0)$ & I $(0 ; 2)$ & $9(7 ; 11)$ & $54(51 ; 57)$ & $36(33 ; 39)$ \\
\hline & \multicolumn{6}{|c|}{ Patient satisfaction with physicians' recommendations about the following topics, $\%(95 \% \mathrm{Cl})$} \\
\hline & Group & $\begin{array}{l}\text { Not at all } \\
\text { satisfied }\end{array}$ & $\begin{array}{l}\text { Somewhat } \\
\text { unsatisfied }\end{array}$ & $\begin{array}{l}\text { Neither satisfied } \\
\text { nor unsatisfied }\end{array}$ & Satisfied & Very satisfied \\
\hline \multirow[t]{2}{*}{ Routine SMBG } & Pat $(n=I, 0 \mid 2)$ & $I(I ; 2)$ & $3(2 ; 4)$ & $18(16 ; 20)$ & $53(49 ; 56)$ & $25(22 ; 28)$ \\
\hline & Phy $(n=974)$ & $0(0 ; 0)$ & $3(2 ; 4)$ & $17(15 ; 20)$ & $71(68 ; 74)$ & $9(7 ; 11)$ \\
\hline \multirow[t]{2}{*}{ Routine medical visits } & Pat $(n=I, 0 \mid 2)$ & $I(0 ; 1)$ & $4(2 ; 5)$ & $17(15 ; 19)$ & $54(51 ; 57)$ & $25(22 ; 27)$ \\
\hline & Phy $(n=974)$ & $0(0 ; 0)$ & I $(0 ; I)$ & $10(8 ; 12)$ & $76(73 ; 79)$ & $14(12 ; 16)$ \\
\hline \multirow[t]{2}{*}{ Diet } & Pat $(n=I, 0 \mid 2)$ & $2(I ; 2)$ & $7(5 ; 8)$ & $26(24 ; 29)$ & $49(46 ; 52)$ & $17(14 ; 19)$ \\
\hline & Phy $(n=974)$ & I $(0 ; 1)$ & $14(12 ; 17)$ & $42(39 ; 45)$ & $39(36 ; 42)$ & $5(3 ; 6)$ \\
\hline \multirow[t]{2}{*}{ Exercise } & Pat $(n=|, 0| 2)$ & $2(1 ; 3)$ & $7(6 ; 9)$ & $28(25 ; 3 \mathrm{I})$ & $45(42 ; 48)$ & $17(15 ; 20)$ \\
\hline & Pat $(n=|, 0| 2)$ & $I(0 ; 1)$ & $15(12 ; 17)$ & $43(40 ; 47)$ & $37(34 ; 40)$ & $4(3 ; 6)$ \\
\hline \multirow[t]{2}{*}{ Oral treatment } & Phy $(n=974)$ & $4(2 ; 5)$ & $4(3 ; 5)$ & $19(17 ; 22)$ & $50(47 ; 53)$ & $24(2 I ; 26)$ \\
\hline & Pat $(n=|, 0| 2)$ & $0(0 ; 0)$ & $0(0 ; I)$ & $9(8 ; 11)$ & $74(7 I ; 77)$ & $16(14 ; 18)$ \\
\hline \multirow[t]{4}{*}{ Injectable treatment } & Phy $(n=974)$ & II $(9 ; 13)$ & $3(2 ; 4)$ & $33(30 ; 36)$ & $37(34 ; 34)$ & $16(13 ; 18)$ \\
\hline & Pat $(n=|, 0| 2)$ & $0(0 ; 1)$ & $9(7 ; 11)$ & $21(18 ; 23)$ & $59(56 ; 62)$ & II (9; I3) \\
\hline & \multicolumn{6}{|c|}{ The extent to which physicians' recommendations allow T2DM control, \% (95\% Cl) } \\
\hline & Group & Not at all & A little & $\begin{array}{l}\text { Neither too much } \\
\text { nor too little }\end{array}$ & Quite a lot & A lot \\
\hline \multirow[t]{2}{*}{ Routine SMBG } & Pat $(n=I, 0 I 2)$ & $2(1 ; 2)$ & $2(1 ; 3)$ & $19(16 ; 21)$ & $55(52 ; 58)$ & $23(20 ; 25)$ \\
\hline & Phy $(n=974)$ & $0(0 ; 1)$ & $5(3 ; 6)$ & $19(16 ; 21)$ & $67(64 ; 70)$ & $9(8 ; 11)$ \\
\hline \multirow[t]{2}{*}{ Routine medical visits } & Pat $(n=|, 0| 2)$ & I $(0 ; 2)$ & $4(3 ; 5)$ & $23(20 ; 25)$ & $53(50 ; 56)$ & $19(17 ; 22)$ \\
\hline & Phy $(n=974)$ & $0(0 ; 0)$ & $I(I ; 2)$ & $14(12 ; 17)$ & 7I (69; 74) & $13(11 ; 15)$ \\
\hline \multirow[t]{2}{*}{ Diet } & Pat $(n=|, 0| 2)$ & $I(0 ; 2)$ & $4(3 ; 6)$ & $22(20 ; 25)$ & $52(49 ; 55)$ & $20(18 ; 23)$ \\
\hline & Phy $(n=974)$ & $0(0 ; 1)$ & $3(2 ; 4)$ & $14(13 ; 17)$ & $51(48 ; 54)$ & $32(29 ; 35)$ \\
\hline \multirow[t]{2}{*}{ Exercise } & Pat $(n=I, 0 \mid 2)$ & $3(2 ; 4)$ & $6(5 ; 7)$ & $27(25 ; 30)$ & $46(42 ; 49)$ & $18(16 ; 20)$ \\
\hline & Phy $(n=974)$ & $0(0 ; 0)$ & $4(2 ; 5)$ & $15(12 ; 17)$ & $51(48 ; 54)$ & $31(28 ; 34)$ \\
\hline \multirow[t]{2}{*}{ Oral treatment } & Pat $(n=|, 0| 2)$ & $7(5 ; 8)$ & $3(2 ; 4)$ & $20(17 ; 22)$ & $49(46 ; 53)$ & $22(19 ; 24)$ \\
\hline & Phy $(n=974)$ & $0(0 ; 0)$ & $0(0 ; 0)$ & $2(1 ; 3)$ & $62(58 ; 65)$ & $36(33 ; 40)$ \\
\hline \multirow[t]{2}{*}{ Injectable treatment } & Pat $(n=|, 0| 2)$ & $22(19 ; 24)$ & $3(2 ; 4)$ & $27(24 ; 30)$ & $33(30 ; 36)$ & $16(14 ; 18)$ \\
\hline & Phy $(n=974)$ & $0(0 ; 0)$ & $I(I ; 2)$ & $4(3 ; 6)$ & $57(54 ; 6 I)$ & $37(34 ; 40)$ \\
\hline
\end{tabular}

Note: $* P<0.001$ in all comparisons.

Abbreviations: $\mathrm{Cl}$, confidence interval; Pat, patients; Phy, physicians; SMBG, self-management of blood glucose; T2DM, type 2 diabetes mellitus.

In general, patients perceived that they "frequently" or "always" followed physicians' recommendations about routine SMBG, routine medical visits, diet, exercise, and oral treatments, while physicians' answers indicated a lower frequency than patients' opinions. As an example, almost a quarter of patients thought they "always" followed physicians' diet (23\%) and exercise recommendations (20\%), while only $3 \%$ and $2 \%$ of physicians believed patients accomplished doctors' suggestions regarding diet and exercise, respectively. Regarding patients' satisfaction with routine SMBG recommendations, the results showed that most patients were "satisfied" or "very satisfied" with them, while 
the majority of physicians indicated that patients were "satisfied" or "neither satisfied nor unsatisfied". Interestingly, physicians perceived lower patient satisfaction with diet (5\%) and exercise (4\%) recommendations compared with the patients $(17 \%)$ themselves. Concerning routine medical visits and oral and injectable treatment, the proportion of patients "satisfied" was lower than the patients satisfaction that physicians perceived.

On the other hand, although most patients and physicians reported that following medical recommendations allowed "quite a lot" of control of T2DM, the proportion was generally higher for physicians. Regarding patients' perceptions about the importance of medical recommendations, most patients $(57 \%)$ reported that it was "important", while most physicians considered it as "very important" (58\%).

\section{Treatment}

Thirty-nine percent of patients reported that physicians "always" take into account comorbidities when they choose a T2DM treatment, while doctors had a more favorable perception, as $70 \%$ of them indicated the same assertion.

The majority of patients $(53 \%)$ perceived that their current T2DM treatment suited their preferences "quite a lot", which was a lower proportion than among the physicians (69\%). Additionally, fewer patients (53\%) than physicians (79\%) believed that their treatment improved their HRQoL "quite a lot". Finally, 46\% of physicians considered new technologies were very important for T2DM management, while only $29 \%$ of patients agreed with this statement.

\section{Discussion}

The findings in this study show that PC physicians and T2DM patients have different perceptions regarding their health status, PROs evaluation, and T2DM management and treatment in routine clinical practice in Spain.

The perceived health status of T2DM patients was worse compared with physicians' opinions; nevertheless, physicians considered that T2DM had a higher impact on patients' health statuses than the patients themselves. This fact is consistent with a previous study showing that health care professionals perceived T2DM as more serious and having a higher negative psychosocial impact than patients did. ${ }^{19}$ In addition, it has been shown that a poor understanding of the disease due to low health care quality may affect patients' perceptions of seriousness, which consequently may influence self-management and treatment adherence; ${ }^{16,19}$ nevertheless, patients' T2DM knowledge was not directly explored in this study and should be considered in future designs.
Regarding PROs, it should be noted that, despite patients and physicians considering PROs assessment important or very important, most patients perceived a low frequency of patient-centeredness in diabetes management and treatment compared with physicians' opinions. In this sense, current guidelines ${ }^{10,13}$ recommend patient-centered care in which patients' needs and preferences regarding treatment should be strongly considered by physicians for clinical decision making. Added to this, PROs assessment is increasingly being used in clinical trials and accepted as a measure of health and well-being in clinical practice. ${ }^{20}$ Nevertheless, results in this study show that the majority of physicians' decisions related to diabetes management and treatment are not completely aligned with patients' opinions and perspectives. On the other hand, it should be remarked that most patients in our study reported lower satisfaction and frequency regarding the information about T2DM, SMBG, and treatment administration techniques provided by the nurses compared with physicians' perceptions. A good interaction between health care professionals (especially nurses) and patients has been shown to be paramount for appropriate patient-centered care ${ }^{15}$ and is related to better disease understanding and patient self-management. ${ }^{16}$ In this regard, the majority of diabetes patients included in this study perceived that they followed medical recommendations regarding routine $\mathrm{SMBG}$, routine medical visits, diet and exercise, and treatment to a greater degree than physicians thought they did.

Given the complexity of diabetes management, T2DM patients need to develop the competency to achieve disease control and improve their outcomes, which requires education, a dietary intervention, exercise management, medication adjustment, and glucose monitoring. ${ }^{10}$ In the Spanish NHS, PC health care professionals, including nurses and physicians, are considered to have the main responsibility for providing information and recommendations to patients about the therapy and self-management of T2DM. ${ }^{21}$ It has been shown that self-management support interventions or programs are effective, ${ }^{22}$ but health care professionals report several limiting factors to providing better health care quality, such as lack of time and resources, inadequate training, or health system problems, ${ }^{21,23}$ that may influence the frequency and quality of education and information interventions.

The study has some limitations inherent to the observational design, including susceptibility to bias and confounding, restricting the ability to define causality, ${ }^{24}$ and the lack of objective outcomes (such as glycemic control) which could allow describing of the patient population in more detail. Additional studies, including data from medical records, 
such as glycemic control and weight variables, could reveal if diabetes could be treated well if patients gave less importance to its management. Moreover, it could be interesting to investigate further how patients' diabetes durations can affect patients' perceptions on diabetes management. In addition, in this study, professionals with at least 5 years' post-residency experience were included, given that the authors wanted to reflect the perspectives of physicians with enough experience in the control of diabetic patients; however, it could be interesting to assess if physicians' experience modifies their perceptions on diabetes management. Although the sample size was calculated to be representative of both sets of participants and the recruitment was performed with the support of independent and unbiased organizations (FEDE and RedGDPs), generalization of results should be performed with caution since participants might not represent the whole Spanish diabetes patient and PC physician population. Finally, considering that the administered questionnaire was not previously validated and that the included patients were from 47 Spanish provinces and the physicians were from 52, the results must be interpreted with caution. The possibility that different perceptions were due to misinterpretation of questions or because of the different perceptions were due to misinterpretation of questions or because of sociodemographics differences between patients and physicians cannot be ruled out.

Despite the described limitations, this study has several strengths, including the large sample of PC physicians and T2DM patients included, which provides relevant information regarding perceptions and views in daily clinical practice of an heterogeneous patients' and physicians' population. These results are evidence of the need of modifying some components of the routine clinical practice in the NHS, such as understanding the patient and physician perspective and specifically incorporating greater patient-centeredness that may lead to the patients' better understanding of the illness and consequently encourage better self-management behaviors.

\section{Conclusion}

The present study gives useful information about both physicians' and T2DM patients' views on PROs assessment, disease management, and treatment, providing useful insight into aspects that may influence medical decision making.

\section{Acknowledgments}

The authors acknowledge FEDE (Federación de Diabéticos Españoles [Spanish Diabetic Patients Federation]) and OUTCOMES'10 for their invaluable contribution to this study.

\section{Disclosure}

Mylan EPD funded the production of this manuscript, which has been read and approved by all named authors. The authors report no other conflicts of interest in this work.

\section{References}

1. International Diabetes Federation (IDF). IDF Diabetes Atlas. 6th ed. Brussels: IDF; 2013. Available from: http://www.idf.org/sites/default/ files/EN_6E_Atlas_Full_0.pdf. Accessed January 8, 2015.

2. Shaw JE, Sicree RA, Zimmet PZ. Global estimates of the prevalence of diabetes for 2010 and 2030. Diabetes Res Clin Pract. 2010;87(1):4-14.

3. Soriguer F, Goday A, Bosch-Comas A, et al. Prevalence of diabetes mellitus and impaired glucose regulation in Spain: the Di@bet.es Study. Diabetologia. 2012;55(1):88-93.

4. Valdés S, Botas P, Delgado E, Díaz Cadórniga F. Mortality risk in spanish adults with diagnosed diabetes, undiagnosed diabetes or prediabetes. The Asturias study 1998-2004. Rev Esp Cardiol. 2009;62(5): 528-534. English, Spanish.

5. DECODE Study Group, European Diabetes Epidemiology Group. Is the current definition for diabetes relevant to mortality risk from all causes and cardiovascular and noncardiovascular diseases? Diabetes Care. 2003;26(3):688-696.

6. Valdés S, Rojo-Martínez G, Soriguer F. [Evolution of prevalence of type 2 diabetes in adult Spanish population]. Med Clin (Barc). 2007; 129(9):352-355. Spanish.

7. Gaede P, Vedel P, Larsen N, Jensen GV, Parving HH, Pedersen O. Multifactorial intervention and cardiovascular disease in patients with type 2 diabetes. $N$ Engl J Med. 2003;348(5):383-393.

8. Crespo C, Brosa M, Soria-Juan A, et al. Costes directos de la diabetes mellitus y de sus complicaciones en España [Direct cost of diabetes mellitus and its complications in Spain (SECCAID Study: Spain estimated cost Ciberdem-Cabimer in Diabetes)]. Av Diabetol. 2013;29:182-189. Spanish.

9. Luscombe FA. Health-related quality of life measurement in type 2 diabetes. Value Health. 2000;3 Suppl 1:15-28.

10. American Diabetes Association. Standards of medical care in diabetes 2014. Diabetes Care. 2014;37 Suppl 1:S14-S80.

11. Bolaños E, Sarría-Santamera A. [Perspective of patients on type-2 diabetes and their relationship with primary care health professionals: a qualitative study]. Aten Primaria. 2003;32(4):195-200. Spanish.

12. Cooper HC, Booth K, Gill G. Patients' perspectives on diabetes health care education. Health Educ Res. 2003;18(2):191-206.

13. Inzucchi SE, Bergenstal RM, Buse JB, et al. Management of hyperglycaemia in type 2 diabetes: a patient-centered approach. Position statement of the American Diabetes Association (ADA) and the European Association for the Study of Diabetes (EASD). Diabetologia. 2012; 55(6):1577-1596.

14. Vinagre I, Mata-Cases M, Hermosilla E, et al. Control of glycemia and cardiovascular risk factors in patients with type 2 diabetes in primary care in Catalonia (Spain). Diabetes Care. 2012;35(4):774-779.

15. Hobbs JL. A dimensional analysis of patient-centered care. Nurs Res. 2009;58(1):52-62.

16. Thomas J 3rd, Iyer NN, Collins WB. Associations between perceived chronic care quality, perceived patient centeredness, and illness representations among persons with diabetes. J Healthc Qual. 2014;36(5):50-59.

17. Population figures at 1 st January 2012 [webpage on the Internet]. Instituto Nacionale de Estadística. Available from: http://www.ine.es/ dynt $3 /$ inebase/en/index.html?padre $=517 \& \mathrm{dh}=1$. Accessed January 8 , 2015. Spanish, English.

18. Perez PB; López-Valcárcel BG, Vega RS. Ministerio de Sanidad, Política Social e Igualdad. Oferta y Necesidad de Especialistas Médicos en España (2010-2025) [Offer and need for medical specialists in Spain] 2011. Available from: http://www.msssi.gob.es/profesionales/formacion/necesidadEspecialistas/doc/11-NecesidadesMEspecialistas(20102025).pdf. Accessed January 8, 2015. Spanish. 
19. Clark M, Hampson SE. Comparison of patients' and healthcare professionals' beliefs about and attitudes towards type 2 diabetes. Diabet Med. 2003;20(2):152-154.

20. Vieta A, Badia X, Sacristán JA. A systematic review of patient-reported and economic outcomes: value to stakeholders in the decision-making process in patients with type 2 diabetes mellitus. Clin Ther. 2011;33(9): 1225-1245.

21. Fonseca M, Fleitas G, Tamborero G, Benejam M, Leiva A. [Lifestyles of primary care physicians: perception and implications on cardiovascular prevention]. Semergen. 2013;39(8):421-432. Spanish.

22. Salinero-Fort MA, Carrillo-de Santa Pau E, Arrieta-Blanco FJ, et al. Effectiveness of PRECEDE model for health education on changes and level of control of $\mathrm{HbAlc}$, blood pressure, lipids, and body mass index in patients with type 2 diabetes mellitus. BMC Public Health. 2011;11:267.
23. Tambo-Lizalde E, Carrasco-Gimeno JM, Mayoral-Blasco S, Rabanaque-Hernández MJ, Abad-Díez JM. [Patient and health professional perceptions on the quality of care provided to diabetic patients]. Rev Calid Asist. 2013;28(2):124-131. Spanish.

24. Yang W, Zilov A, Soewondo P, Bech OM, Sekkal F, Home PD. Observational studies: going beyond the boundaries of randomized controlled trials. Diabetes Res Clin Pract. 2010;88 Suppl 1:S3-S9.

\section{Publish your work in this journal}

Patient Preference and Adherence is an international, peer-reviewed, open access journal that focuses on the growing importance of patient preference and adherence throughout the therapeutic continuum. Patient satisfaction, acceptability, quality of life, compliance, persistence and their role in developing new therapeutic modalities and compounds to optimize clinical outcomes for existing disease states are major areas of interest for the journal. This journal has been accepted for indexing on PubMed Central. The manuscript management system is completely online and includes a very quick and fair peer-review system, which is all easy to use. Visit http://www dovepress.com/testimonials.php to read real quotes from published authors.

Submit your manuscript here: http://www.dovepress.com/patient-preference-and-adherence-journal 